\title{
Superscaling and Charge-changing Neutrino Cross Sections
}

\author{
M.B. Barbaro, J.E. Amaro, J.A. Caballero, T.W. Donnelly, A. Molinari, I. Sick a \\ ${ }^{a}$ Dip. di Fisica Teorica, Univ. di Torino and INFN, Via P. Giuria 1, I10125 Turin, Italy, \\ Dep. de Física Moderna, Univ. de Granada, 18071 Granada, Spain, \\ Dep. de Física Atómica, Molecular y Nuclear, Univ. de Sevilla, Apdo. 1065, 41080 Sevilla, Spain, \\ CTP, LNS and Dep. of Physics, Massachusetts Institute of Technology, Cambridge, MA 02139, USA, \\ Dep. für Physik und Astronomie, Univ. Basel, CH-4056 Basel, Switzerland
}

High-quality predictions for neutrino-nucleus cross sections are needed for use in on-going experimental studies of neutrino oscillations at $\mathrm{GeV}$ energies, where a fully relativistic treatment of the neutrino-nucleus scattering is mandatory, but hard to achieve. Of course any reliable calculation for neutrino scattering should first be tested against electron scattering in the same kinematical conditions. In fact, while relativistic modeling of the nuclear dynamics is capable of getting the basic size and shape of the inclusive cross section, it can hardly account for important details of the response. Specifically, the data display a long tail which is largely absent in most modeling, possibly due to the absence of classes of short-range correlation effects and to the treatment of final-state interactions.

In order to overcome these difficulties, instead of using a specific nuclear model, we propose here a method for extracting informations on the nuclear dynamics from electron scattering experiments and use them to predict the neutrinonucleus cross section.

The approach is based on the superscaling behavior of the electron-nucleus cross section in both the quasi-elastic (QE) and $\Delta$ peaks. Detailed studies of superscaling can be found in Refs. 12] here we simply recall the basic idea. By dividing the experimental inclusive cross section for various momentum transfers $q$ and nuclei (or, equivalently, Fermi momentum $k_{F}$ ) by an appropriate single-nucleon cross section, a reduced cross section $f$, called the superscaling function, is obtained, which embodies the nuclear dynam- ics. This can be plotted versus a well-chosen scaling variable: if no dependence of $f$ upon $q$ is observed, we call this behavior scaling of the first kind, whereas if no dependence occurs on the specific nucleus, we call it scaling of the second kind; if both types of scaling behavior are found we say that superscaling occurs.

Several choices for the appropriate scaling variable have been proposed in the literature. Here we choose that which naturally emerges from the relativistic Fermi gas (RFG) model. In this framework the nuclear response functions in both the quasi-elastic $(X=Q E)$ and $\Delta$-resonance $(X=\Delta)$ regions have the general structure

$R_{X}=\frac{\mathcal{N} m_{N}}{q k_{F}}\left[R_{X}\right]^{s . n .} f_{R F G}^{X}\left(\psi_{X}\right)$

where $\mathcal{N}$ is the appropriate nucleon number and $\left[R_{X}\right]^{s . n \text {. }}$ is the single-nucleon response function. The RFG superscaling function

$f_{R F G}^{Q E}(\psi)=f_{R F G}^{\Delta}(\psi)=\frac{3}{4}\left(1-\psi^{2}\right) \theta\left(1-\psi^{2}\right)$

is independent of $q$ and $k_{F}$, being a function of a specific combination of $q, \omega$ and $k_{F}$, the scaling variable, defined as

$\psi_{X}= \pm \sqrt{\frac{1}{T_{F}}\left(\frac{q}{2} \sqrt{\rho_{X}^{2}+1 / \tau}-\frac{\omega}{2} \rho_{X}-m_{N}\right)}$

where $T_{F}$ is the Fermi kinetic energy, $4 m_{N}^{2} \tau=$ $q^{2}-\omega^{2}$ and $\rho_{X}=1+\left(m_{X}^{2}-m_{N}^{2}\right) /\left(q^{2}-\omega^{2}\right)\left(m_{Q E}\right.$ being the nucleon mass). The sign in Eq. (3) is $+(-)$ if the energy transfer $\omega$ is higher (lower) than the one corresponding to the maximum of 
the peak. Moreover, a small energy shift is introduced, implying $\psi \rightarrow \psi^{\prime}$, in order to force the maximum of the response to occur for $\psi_{X}^{\prime}=0$.

At sufficiently high energies the electron scattering data exhibit both types of scaling behavior in the quasi-elastic region. For specific nuclei the first-kind scaling is quite good at excitation energies below the QE peak (the so-called scaling region): this is the familiar $y$-scaling behavior. At energies above the peak, where nucleon resonances (especially the $\Delta$ ) are important, this type of scaling is broken. The scaling violations apparently reside in the transverse response, but not in the longitudinal, which appears to superscale. In fact, this is not unexpected, since MEC and inelastic contributions, which do not scale, are predominantly transverse in the kinematic regions of interest. On the other hand scaling of the second kind works very well in the scaling region and, even in the resonance region, is only violated at roughly the $20 \%$ level.

The approach taken in 22 therefore has been to use the experimental longitudinal responses to define the superscaling function. Reliable separations of data into their longitudinal and transverse contributions for $A>4$ are available only for a few nuclei; all of these response functions have been used to extract the "universal" quasielastic response function $f^{Q E}$ and to obtain a parameterization by a simple function. Fig. 1 (upper panel) shows $f^{Q E}\left(\psi_{Q E}^{\prime}\right)$ averaged over the nuclei employed, together with the corresponding fit, plotted against the scaling variable $\psi_{Q E}^{\prime}$.

Note that $f^{Q E}\left(\psi_{Q E}^{\prime}\right)$ has a somewhat asymmetric shape and a tail that extends towards positive values of $\psi_{Q E}^{\prime}$. In contrast, the RFG superscaling function (3) is symmetric, is limited strictly to the region $-1 \leq \psi_{Q E}^{\prime} \leq+1$ and has a maximum value of $3 / 4$, while the empirical superscaling function reaches only about 0.6. The microscopical origin of this asymmetric shape has been the object of recent investigations in the context of the relativistic impulse approximation [3]. Let us now focus on the resonance region. In order to isolate the $\Delta$ contribution, we subtract from the total experimental cross sections the quasi-elastic cross section recalculated
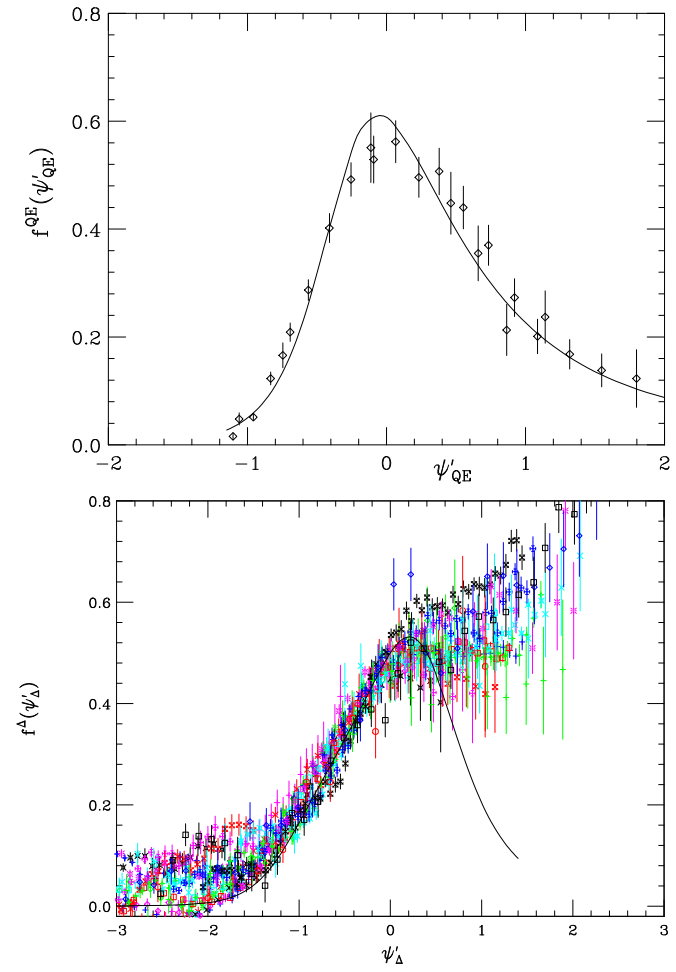

Figure 1. Experimental $\mathrm{QE}$ and $\Delta$ superscaling functions versus the corresponding scaling variables

using the universal $f^{Q E}\left(\psi_{Q E}^{\prime}\right)$ introduced above. That is, we remove the impulsive longitudinal and transverse contributions that arise from elastic $e N$ scattering, leaving (at least) MEC effects with their associated correlations and impulsive contributions arising from inelastic eN scattering. The MEC effects can be ignored as they provide relatively small corrections [4, and thus this yields a response which is largely dominated by the $\Delta$, at least for energy losses lower than the maximum of the $\Delta$ peak.

Using the same procedure as in the QE peak, we can again reduce the left-over cross section by dividing by the appropriate single-nucleon $N \rightarrow$ $\Delta$ cross section and display the result versus the scaling variable $\psi_{\Delta}^{\prime}$ defined in Eq. (3). In Fig. 1 (lower panel) we show the resulting $f^{\Delta}\left(\psi_{\Delta}^{\prime}\right)$ extracted from the high-quality world data for inclusive electron scattering from ${ }^{12} \mathrm{C}$ and ${ }^{16} \mathrm{O}$ in the $\mathrm{QE}$ and $\Delta$ regions. These data span energies 
extending from $300 \mathrm{MeV}$ to $4 \mathrm{GeV}$ and scattering angles from 12 to 145 degrees, depending on the beam energy. As for $f^{Q E}$, the experimental values of $f^{\Delta}$ have been parameterized by a simple analytical function (solid line). The data appear to scale reasonably well up to the peak of the $\Delta$ $\left(\psi_{\Delta}^{\prime} \cong 0\right)$, although clearly for still higher excitation energies the scaling is broken by processes that are not well represented via $\Delta$-dominance (other resonances and, at the larger values of $q$, the tail of deep inelastic scattering). There is also some excess at large negative $\psi_{\Delta}^{\prime}$ which breaks the scaling to some degree, probably due to contributions from MEC and their associated correlations 4 .

The above analysis can now be checked by assembling all of the pieces obtained via the scaling procedures to produce a total inclusive cross section which can be compared with data. Several examples of such a comparison are shown in Ref. [5]. The answers are very encouraging, and only for specific kinematics do we see deviations as large as $10-20 \%$, probably due to MEC and correlations. In particular the dip region, usually hard to get from microscopic models, is nicely fitted in the present context.

We are then in a position to take a step in a new direction. Since the above superscaling functions, upon being multiplied by the electromagnetic $N \rightarrow N$ and $N \rightarrow \Delta$ single-nucleon cross sections, reproduce quite well the total nuclear electromagnetic cross section, we can just as well multiply by the corresponding charge-changing weak interaction $N \rightarrow N$ and $N \rightarrow \Delta$ singlenucleon cross sections to obtain predictions for neutrino reactions in nuclei at similar kinematics. The result for the reaction ${ }^{12} \mathrm{C}\left(\nu_{\mu}, \mu^{-}\right)$is shown in Fig. 2 versus the muon momentum $k^{\prime}$ for typical kinematical conditions. Cross sections at different kinematics and for antineutrino scattering can be found in Ref. [5. As discussed above, the predictions at momenta to the left of the $\Delta$ peak (excitations lying above the $\Delta$ region) are unreliable, since our scaling approach does not fully account for meson production, including resonances other than the $\Delta$, and DIS processes. For comparison, in Fig. 2 we also show the cross section obtained using the RFG. Modeling based on

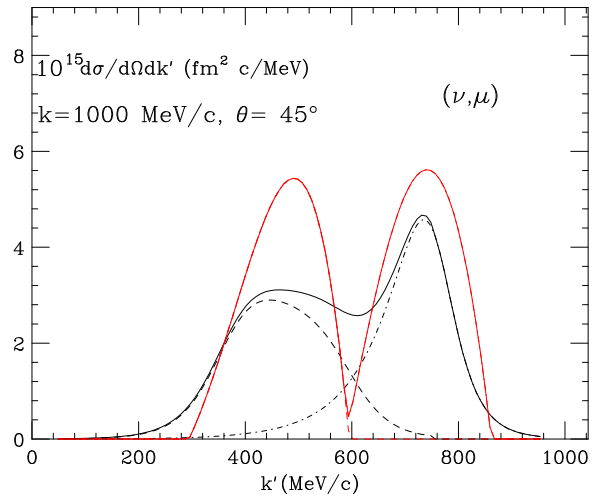

Figure 2. $\nu_{-}{ }^{12} \mathrm{C}$ cross section plotted versus the muon momentum $k^{\prime}$. Dash-dotted: QE; dashed: $\Delta$; solid: total; heavier lines (red on-line): RFG.

mean-field theory where finals state interactions are not included is very similar to the RFG result, since at the relatively high energies of interest the dynamical effects embodied in an effective mass are expected to be small. Likewise, relativized shell model predictions are close to the RFG predictions [6] and, moreover, RPA correlations are expected to be relatively small for the high energies involved. Thus, the RFG predictions effectively represent a larger set of models. As can be seen in the figure, all therefore differ significantly from the scaling predictions.

In conclusion, given the success of the scaling approach in studies of inclusive electron scattering for the kinematic region under study, we expect neutrino reaction cross sections also obtained using scaling ideas to be more robust than those based directly on existing models.

\section{REFERENCES}

1. W.M.Alberico et al., PRC 38, 1801 (1988); M.B.Barbaro et al., NPA 643, 137 (1998); C.Maieron et al., PRC 65, 025502 (2002).

2. D.B.Day et al., Ann. Rev. Nucl. Part. Sci. 40, 357 (1990); J.Jourdan, NPA 603, 117 (1996);

T.W.Donnelly et al. PRC 60, 065502 (1999).

3. J.A.Caballero et al., arXiv:nucl-th/0504040

4. J.E.Amaro et al., Phys. Rep. 368, 317 (2002) and references therein.

5. J.E.Amaro et al., PRC 71, 015501 (2005).

6. J.E.Amaro et al., PRC 71, 065501 (2005). 